\title{
Cortical versus Pial Venous Drainage in Dural Arteriovenous Fistula
}

\author{
Sang Hun Lee, MD, Dae Chul Suh, MD
}

It is difficult to say that cortical venous reflux is directly related with intracerebral hemorrhage in dural arteriovenous fistula (DAVF) because the cortical vein anatomically exists in the subarachnoid space [1]. Hemorrhage is the most serious event in cranial DAVF. Cortical venous reflux (CVR) has been known as a prognostic risk factor for hemorrhage in cranial DAVF [2]. Hemorrhage risk is related to lesion location, sex and cortical venous reflux [3]. Venous drainage pattern, especially the presence of cortical venous reflux, is a significant factor predicting intracranial hemorrhage from DAVF [4]. Annual hemorrhage rate of cranial DAVF is about $5 \%$ when there is cortical venous reflux.

However, terminology of CVR is not exactly defined because meningeal venous reflux is also used in some literature [5]. At this present, there is a need of exact anatomical discrimination between cortical and pial venous refluxes (PVR) [1]. Veins were defined as "cortical" when they coursed along the cortical surface

Key Words : Dural arteriovenous fistula; Pial venous drainage; Pial venous reflux; Cortical venous drainage; Cortical venous reflux

\footnotetext{
All authors: Departments of Radiology, Asan Medical Center, University of Ulsan College of Medicine, Seoul, South Korea Received February 16, 2017;

accepted after revision February 28, 2017.

Correspondence to: Dae Chul Suh, MD, PhD, Department of Radiology, Asan Medical Center, University of Ulsan, 88, Olympicro, 43-gil, Songpa-gu, Seoul 05505, South Korea

Tel. 82.2.3010.4366 Fax. 82.2.3010.0090

E-mail: dcsuh@amc.seoul.kr

This is an Open Access article distributed under the terms of the Creative Commons Attribution Non-Commercial License (http://creativecommons.org/licenses/by-nc/3.0) which permits unrestricted non-commercial use, distribution, and reproduction in any medium, provided the original work is properly cited.
}

draining into the venous sinus and as "pial" when the fine and tortuous veins were within the brain or on the brain surface under the pial membrane. Presence of PVR was also decided by comparison with cortical veins in the venous phase of the ipsilateral internal carotid arteriogram. Compared with cortical veins, which are regarded as the main leptomeningeal veins draining into sinus, the fine pial veins or the intracortical veins beneath the pial membrane were regarded as having a corkscrew-like appearance or intraparenchymal course, which cannot be seen on a routine normal angiogram $[1,7]$.

Cavernous sinus dural arteriovenous fistula (CSDAVF) which seems to occur more commonly in Korea is different in several aspects from DAVF involving other dural sinuses [8]. Anatomically, the cavernous sinus is an extradurally located sinus, whereas other dural sinuses are located between two dural walls in the cranial cavity. In addition, the clinical presentations are known to be benign because cavernous sinus has sufficient venous drainage routes; these include the superior ophthalmic vein, inferior and superior petrosal sinuses, superficial middle cerebral vein, and the coronary sinus to the opposite side of cavernous sinus. Therefore, cortical and pial venous refluxes from the cavernous sinus seem to be less often compared to other type of lesion. Thus, CSDAVF which presents with various symptoms patterns and may have different pathophysiology and requires diverse treatment strategies [8-10]. It is also interesting to note that higher prevalence of diabetes mellitus was found in more aggressive type of DAVF. In addition, patients with aggressive symptoms had a higher prevalence of diabetes mellitus [11].

Brain edema and hemorrhage were the similarly associated with pial venous reflux since the concept of 

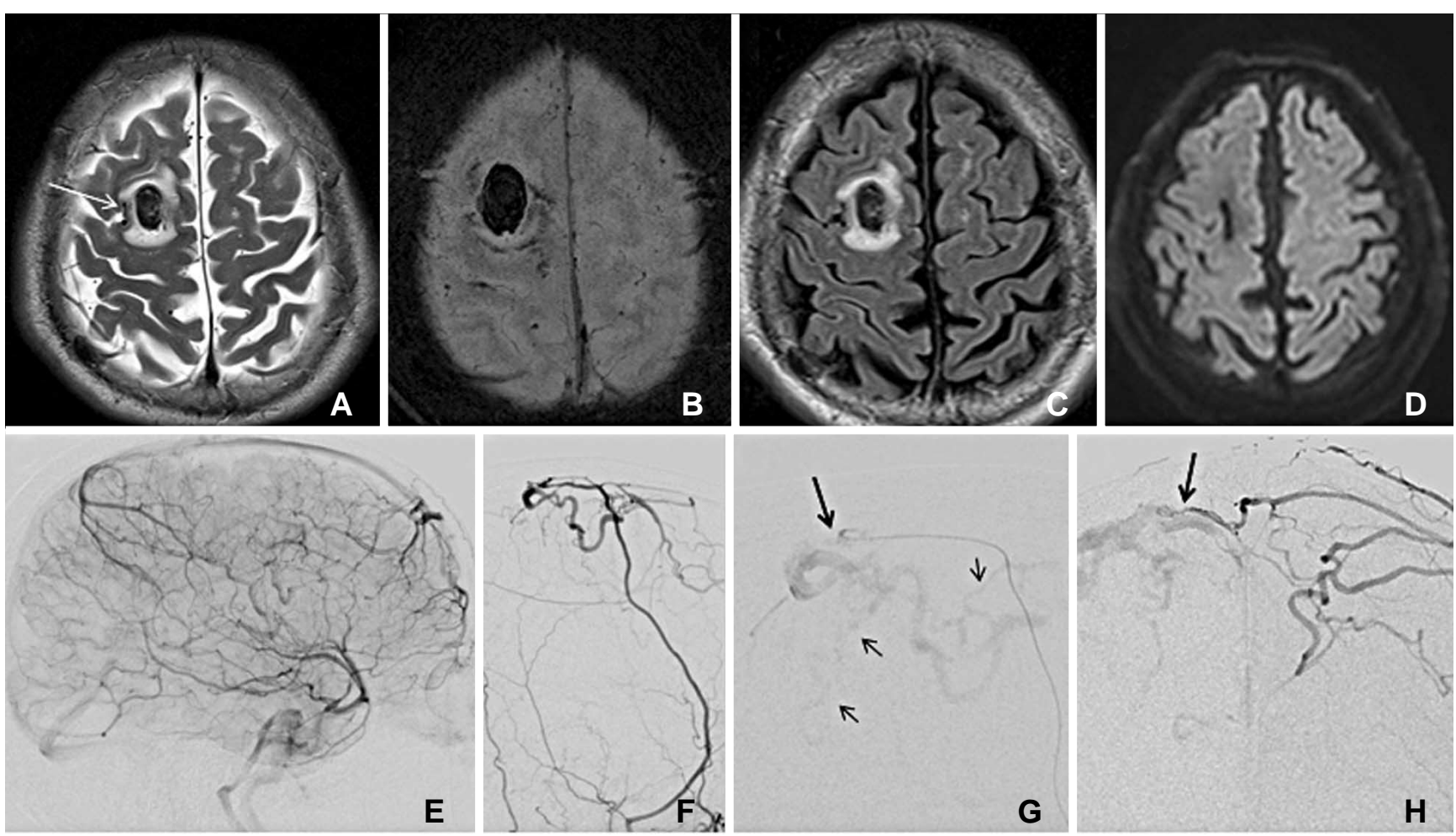

Fig. 1. A 75-year-old male presented with seizure. He had hypertension and diabetes mellitus for 5 years. (A) T2WI shows a focal hemorrhage surrounded by an edema in right frontal lobe. Note a small vein at the brain surface (white arrow) (B) Susceptibility-weighted image shows increased subsceptibility around the hemorrhage due to the engorged pial veins. (C) FLAIR image shows edema and hemorrhage. (D) Thirteen months follow-up FLAIR image after successful embolization reveals resolved hemorrhage and edema. (E) Venous phase of the right internal carotid arteriogram shows the patent superior sagittal sinus but non-filling of the parietal cortical veins. (F) The right internal maxillary arteriogram shows a dural arteriovenous fistula supplied by the middle meningeal artery. (G) Selective angiogram at the distal middle meningeal artery (large arrow) shows filling of the pial veins (small arrows). (H) Contralateral internal maxillary arteriogram shows a filling of the same fistula from the opposite side (arrow).

venodural junction and venopial junction was raised. Subarachnoid space anatomically exists between venoudural and venopial junction. Therefore venous drainage into the pial vein occurs via cortical vein or sometimes directly into pial veins. Cerebral edema can developed into hemorrhage within several weeks in those cases with direct pial venous reflux (Fig. 1).

In our previous study showed that PVR is more closely related to hemorrhage or edema [1]. PVR was associated with hemorrhage or edema in $75 \%$ of patients and was related to a certain brain area, whereas CVR occurred in a wide vascular territory [1]. In conclusion, PVR is more closely associated with the hemorrhage or edema than CVR in patients with DAVF. PVR can occur not only as a part of CVR but also directly in certain types of DAVF.

\section{References}

1. Zhao LB, Suh DC, Lee DG, Kim SJ, Kim JK, Han S, et al. Association of pial venous reflux with hemorrhage or edema in dural arteriovenous fistula. Neurology 2014;82:1897-1904

2. Cognard C, Gobin YP, Pierot L, Bailly AL, Houdart E, Casasco A, et al. Cerebral dural arteriovenous fistulas: clinical and angiographic correlation with a revised classification of venous drainage. Radiology 1995;194:671-680

3. Kirsch M, Liebig T, Kuhne D, Henkes H. Endovascular management of dural arteriovenous fistulas of the transverse and sigmoid sinus in 150 patients. Neuroradiology 2009;51:477-483

4. Peng T, Liu A, Jia J, Jiang C, Li Y, Wu Z, et al. Risk factors for dural arteriovenous fistula intracranial hemorrhage. J Clin Neurosci 2014;21:769-772

5. Borden JA, Wu JK, Shucart WA. A proposed classification for spinal and cranial dural arteriovenous fistulous malformations and implications for treatment. J Neurosurg 1995;82:166-179

6. Marin-Padilla M, Knopman DS. Developmental aspects of the intracerebral microvasculature and perivascular spaces: insights into brain response to late-life diseases. J Neuropathol Exp Neurol 2011;70:1060-1069

7. Willinsky R, Terbrugge K, Montanera W, Mikulis D, Wallace MC. Venous congestion: an MR finding in dural arteriovenous malformations with cortical venous drainage. AJNR Am J Neuroradiol 1994;15:1501-1507

8. Chung SJ, Kim JS, Kim JC, Lee SK, Kwon SU, Lee MC, et al. Intracranial dural arteriovenous fistulas: analysis of 60 patients. 


\section{Sang Hun Lee, et al.}

Cerebrovasc Dis 2002;13:79-88

9. Choi BS, Park JW, Kim JL, Kim SY, Park YS, Kwon HJ, et al. Treatment Strategy Based on Multimodal Management Outcome of Cavernous Sinus Dural Arteriovenous fistula (CSDAVF). Neurointervention 2011;6:6-12

10. Suh DC, Lee JH, Kim SJ, Chung SJ, Choi CG, Kim HJ, et al. New concept in cavernous sinus dural arteriovenous fistula: correlation with presenting symptom and venous drainage patterns. Stroke 2005;36:1134-1139

11. Lee DG, Zhao LB, Shim JH, Lee DH, Suh DC. Relationship between diabetes mellitus with dural arteriovenous fistula. Neuroradiology 2013;55:1129-1134 